\title{
Delayed Liver Function Impairment Secondary to Interferon $\beta-1 a$ Use in Multiple Sclerosis
}

\author{
Ming-Feng Liao ${ }^{a, d}$ Su-Chen Yen ${ }^{b, d}$ Chun-Yen Lin ${ }^{c, d}$ Rong-Kuo Lyu ${ }^{a, d}$ \\ ${ }^{a}$ Department of Neurology, ${ }^{b}$ Department of Pharmacy, Clinical Pharmacy, and \\ 'Department of Hepato-Gastroenterology, Chang Gung Memorial Hospital, Linkou, and \\ ${ }^{d}$ College of Medicine, Chang-Gung University, Taipei, Taiwan, ROC
}

\section{Key Words}

Multiple sclerosis · Hepatitis · Liver function impairment $\cdot$ Interferon $\beta$. Azathioprine

\begin{abstract}
Interferon $\beta-1 a$ is a widely used immunomodulation treatment for multiple sclerosis. Liver function impairment is a common side effect and usually develops in the first 6 months after interferon use. Here, we describe 2 multiple sclerosis patients who developed delayed liver function impairment 5 years after receiving interferon $\beta$-1a treatment. Their liver function recovered after discontinuing interferon use, and further detailed hepatological evaluations excluded other etiologies of liver function impairment. Our case reports illustrate that liver function impairment induced by interferon $\beta-1 a$ can be delayed for 5 years after starting treatment and, probably, this is an idiosyncratic reaction. Regular liver function monitoring in multiple sclerosis patients who receive interferon $\beta$ is necessary even after the first 6 months of treatment, especially in those patients with concomitant use of other liver-toxic medications.
\end{abstract}

\section{Introduction}

Interferon $\beta-1 \mathrm{a}$ is a commonly used immunomodulation treatment for multiple sclerosis. It has been proven that interferon $\beta-1$ a can reduce relapse rate, disease activity [measured by magnetic resonance imaging (MRI) scanning], and prevent disease progression [1-3]. Common side effects of interferon $\beta$-1a include flu-like symptoms, headache, depression, insomnia, inflammation or cutaneous necrosis at the injection site [1-3]. Laboratory abnormalities, including liver function impairment, were also commonly 
observed in patients receiving interferon treatment [1-6]. The incidence of abnormal liver function tests ranged from 1 to $67 \%$ in different studies [2-6]. In most cases, liver enzyme elevation developed during the first 6 months after receiving interferon; therefore, regular liver function monitoring in the first 6 months is recommended $[2,4-6]$. Here, we report 2 multiple sclerosis patients with interferon $\beta$-1a treatment who developed delayed liver function impairment around 5 years after interferon $\beta$-1a treatment.

\section{Case Reports}

\section{Patient 1}

A 56-year-old Taiwanese woman was diagnosed as having multiple sclerosis in May 2000. Her first attack presented as left optic neuritis in December 1998. Her second attack, with brain stem encephalitis, developed in May 2000. Cranial MRI studies showed T2weighted hyperintense lesions in the inferior medulla and upper cervical spinal cord at C1 level at that time. Her IgG index was 0.77 (reference $<0.5$ ), but oligoclonal bands were not found in the cerebrospinal fluid. Autoimmune factors such as anti-SSA/SSB antibody, antinuclear antibody, and rheumatoid factor were within the normal ranges. She was diagnosed as having multiple sclerosis and received interferon $\beta-1$ a (Rebif $44 \mu \mathrm{g}$ subcutaneous injection 3 times a week) starting in October 2002. She had sensations of chill after interferon use at first, but could tolerate it. The results of liver function tests in March 2005 were still within the normal range: alanine transaminase (ALT) $13 \mathrm{U} / \mathrm{l}$ [upper limit of normal (ULM) $<36 \mathrm{U} / \mathrm{l}]$. Recurrent thoracic myelopathy developed in March 2006. The thoracic MRI studies showed T2-weighted hyperintense lesions in the central gray matter around the T5T6 level (two segments). However, her anti-aquaporin 4 (AQP4) antibody test result was negative (the manufacturer's recommended level for seropositivity was $>5 \mathrm{U} / \mathrm{ml}$; assessed by Elisa RSR AQP4 Ab kits; provided by Kronus, Ltd.). She was still diagnosed as having multiple sclerosis rather than neuromyelitis optica (NMO) according to Wingerchuk et al.'s [7] criteria for NMO in 2006 and McDonald's diagnostic criteria for multiple sclerosis in 2010 [8], and she continued to receive interferon therapy. After the thoracic myelitis, mycophenolate prednisolone and cyclophosphamide were also used to control her disease. However, she could not tolerate the side effects of these medications. Her medications were changed to azathioprine $50 \mathrm{mg}$ daily in December 2006. During this period, she also received carbamazepine $100 \mathrm{mg}$ twice a day. Follow-up liver function tests in October 2007 then showed impaired liver function: aspartate transaminase (AST) $93 \mathrm{U} / \mathrm{l}[2.7 \times \mathrm{ULM}(<34 \mathrm{U} / \mathrm{l})]$ and ALT $100 \mathrm{U} / \mathrm{l}(2.8 \times \mathrm{ULM})$. She only had mild general malaise without other symptoms such as jaundice. She did not consume any alcohol and denied taking any over-the-counter medication. However, she took some Chinese herbs with unknown content sometimes. Initially, azathioprine was suspected to induce liver function impairment, and the dose was reduced to $25 \mathrm{mg}$ daily in December 2007 and further reduced to $25 \mathrm{mg}$ every other day in January 2008. However, her liver function gradually worsened in the next few months. In May 2008, the results of her liver function tests were as follows: AST $213 \mathrm{U} / \mathrm{l}(6.3 \times \mathrm{ULM})$; ALT $282 \mathrm{U} / \mathrm{l}(7.8 \times \mathrm{ULM})$; alkaline phosphatase $($ ALK-P) $145 \mathrm{U} / \mathrm{l}[1.5 \times \mathrm{ULM}(<94 \mathrm{U} / \mathrm{l})]$; total bilirubin $0.7 \mathrm{mg} / \mathrm{dl}$ (ULM $<1.3 \mathrm{mg} / \mathrm{dl}$ ). Detailed hepatological evaluation was started. Results of hepatitis markers (HBsAg and anti-HCV antibody) and autoimmune titers (antinuclear antibody, anti-smooth muscle antibody, and anti-mitochondrial antibody) were all within the normal ranges. In addition, she did not have any other drug reactions such as renal insufficiency, hemolytic anemia, leucopenia, and thrombocytopenia besides liver function impairment. Drug-induced hepatitis was highly suspected. Interferon $\beta-1$ a was reduced to 
$44 \mu \mathrm{g}$ twice a week; carbamazepine and azathioprine were discontinued in May 2008. She also stopped taking Chinese herbs after that time. Her liver function improved after reducing the interferon dose and discontinuing other concomitant medications [July 2008: AST $51 \mathrm{U} / \mathrm{l}$ $(1.5 \times$ ULM); ALT 80 U/l (2.2 × ULM); r-glutamyl transpeptidase (r-GT) 110 U/l (ULM <71 $\mathrm{U} / \mathrm{l})]$. Then, her liver function returned into normal ranges after interferon was discontinued in August 2008 [January 2009: AST 23 U/l; ALT 27 U/l; r-GT 75 U/l (1.06 × ULM)]. After a period of follow-up, interferon $\beta$-1a (22 $\mu \mathrm{g}$ twice a week) and carbamazepine (100 mg twice a day) were added again in March 2009. However, her liver enzymes were elevated again [April 2009: AST 43 U/l (1.3 × ULM); ALT 69 U/l $(1.9 \times$ ULM); r-GT 436 U/l $(6.1 \times$ ULM)] a month later. So, interferon was discontinued, while carbamazepine treatment was continued (100 mg twice daily). Her liver enzymes returned to normal ranges [AST $24 \mathrm{U} / \mathrm{l}$; ALT $31 \mathrm{U} / \mathrm{l}$; r-GT $150 \mathrm{U} / \mathrm{l}(2.1 \times \mathrm{ULM})]$ again in May 2009. The results of her liver function test remained in the normal ranges at follow-up (November 2010: AST $18 \mathrm{U} / \mathrm{l}$; ALT $19 \mathrm{U} / \mathrm{l}$ ). The Roussel Uclaf Causality Assessment Method (RUCAM) scale for this patient after receiving interferon treatment is 8 [9].

\section{Patient 2}

A 43-year-old Taiwanese woman had right optic neuritis in 2001. Her second attack, with brain stem encephalitis, developed in April 2007. Cranial MRI studies at that time showed multiple periventricular and right paramedian pontine white matter lesions which fitted the criteria of dissemination in space according to the McDonald criteria [8]. Her IgG index was 0.65; oligoclonal bands were not found in the cerebrospinal fluid. Autoimmune factors such as anti-SSA/SSB antibody, antinuclear antibody, and rheumatoid factor were within the normal ranges. Since she did not have acute myelitis, which is one of the absolute diagnostic criteria of NMO [7], she did not receive an anti-AQP4 antibody test. She received interferon $\beta$-1a (Rebif $44 \mu$ g subcutaneous injection 3 times a week) under the diagnosis of multiple sclerosis starting in January 2007. Results of liver function tests in November 2007 were within the normal ranges: ALT $33 \mathrm{U} / \mathrm{l}$ and AST $22 \mathrm{U} / \mathrm{l}$. Concomitant medications during this period were baclofen $10 \mathrm{mg}$ twice a day and zolpidem $10 \mathrm{mg}$ before sleep. However, follow-up liver function tests in July 2012 showed impaired liver function: AST $93 \mathrm{U} / \mathrm{l}$ (6.8 $\times$ ULM); ALT $251 \mathrm{U} / \mathrm{l}(7.4 \times \mathrm{ULM})$; amylase 636 [6.4 × ULM $(<100 \mathrm{U} / \mathrm{l})]$; r-GT $73 \mathrm{U} / \mathrm{l}$, and total bilirubin $0.8 \mathrm{mg} / \mathrm{dl}$. She did not have any clinical symptoms like malaise or jaundice. She also denied any alcoholic intake and taking any other medication during this period. Rebif was reduced to $22 \mu \mathrm{g} 3$ times a week. Detailed hepatological evaluation was also started. Results of hepatitis markers (HBsAg and anti-HCV antibody) and autoimmune titers (antinuclear antibody and anti-mitochondrial antibody) were within normal ranges. She only had mild elevated anti-smooth muscle antibody tilter (1:80). Further abdominal computed tomography scan studies only showed a slight predominance of periportal fat raising suspicion of inflammatory change. Her liver function improved a little after reducing the interferon dose [November 2012: AST $84 \mathrm{U} / \mathrm{l}(2.5 \times \mathrm{ULM})$; ALT $92 \mathrm{U} / \mathrm{l}(2.6 \times \mathrm{ULM})]$. Interferon was discontinued in January 2013 due to the persistently elevated liver function tests. The results of her liver function test then returned into normal ranges (February 2013: AST 35 $\mathrm{U} / \mathrm{l}$; ALT $36 \mathrm{U} / \mathrm{l}$ ), and interferon treatment was not resumed this time. The RUCAM scale for this patient after receiving interferon treatment is 7. 
Liao et al.: Delayed Liver Function Impairment Secondary to Interferon $\beta$-1a Use in Multiple Sclerosis

\section{Discussion}

Both our patients were diagnosed as having multiple sclerosis and both had elevated liver function tests around 5 years after starting interferon $\beta$-1a treatment. Patient 1 had some concomitant medication use, namely carbamazepine and azathioprine. Her liver function improved after reducing the interferon dose, turning back into normal ranges after discontinuing interferon and concomitant medications. Since the liver function impairment after interferon use is dose dependent $[4,6]$, we monitored liver function tests after rechallenge with low-dose interferon. Liver function impairment developed again after rechallenge with low-dose interferon, and liver function became normal again after discontinuation of interferon. Detailed laboratory tests excluded any other etiology of liver function impairment; other concomitant drug effects were excluded by the secondary low-dose interferon re-challenge test. Patient 2 developed liver function impairment without other concomitant hepatotoxic medications. Her liver function tests improved after reducing interferon dose, returning into normal ranges after discontinuing interferon treatment. Although we did not initiate re-challenge with low-dose interferon, detailed laboratory tests also excluded any other etiology of liver function impairment. The RUCAM scales after interferon treatment in these two patients are 8 and 7, respectively, which indicated that liver function impairment caused by interferon $\beta$-1a was probable.

Most studies showed that abnormal liver function after interferon $\beta$-1a treatment developed in the first 6 months after receiving interferon $[2,4,6]$. In a study of $>1,000$ patients who received interferon $\beta$-1a treatment, $75 \%$ of liver function impairment developed within the first 6 months after starting therapy, and regular liver function monitoring is suggested only during the first 6 months of treatment [4]. Delayed liver function impairment is rare in the literature review. Only Christopher et al. [10] report a multiple sclerosis patient with delayed liver function impairment 3 years after interferon $\beta$ treatment. Our patients quite unusually developed liver function abnormalities 5 years after starting interferon therapy.

Most multiple sclerosis patients on interferon treatment who had elevated liver function tests were usually asymptomatic and mild cases. Elevated liver function tests rarely led to interferon discontinuation [1,3]. The liver enzymes usually return into normal ranges gradually after dose reduction or interruption of therapy $[3,4]$. A high dose and high frequency of interferon $\beta$ use may increase the risk of liver function impairment in patients with multiple sclerosis $[4,6]$. Liver biopsies of these patients usually reveal portal inflammation with centrivenular hemorrhage and mononuclear cell infiltration, which is consistent with acute hepatitis [11]. If extreme liver enzyme elevation $(>20 \times$ ULM) or jaundice develops, interferon should be discontinued immediately; if liver enzyme elevation is mild ( $>5$ and $<20 \times$ ULM), the interferon dose should be reduced [4]. We tried to reduce the interferon dose in our 2 patients at first. However, they still had liver enzyme elevation even on low-dose interferon. Therefore, interferon treatment was discontinued, and the liver enzymes returned into normal ranges.

Concomitant medications with interferon therapy, including acetaminophen, propionic acid derivatives like ibuprofen, non-selective monoamine oxidase inhibitors, selective serotonin re-uptake inhibitors, baclofen, opioid, anti-epileptic medications, and cyclooxygenase inhibitors, have been studied; there was no evidence that concomitant use of these medications will increase the risk of liver function impairment [4]. However, patient 1 had taken azathioprine, which has potential hepatic toxicity, at the same time [12]. A previous report showed that $33 \%(5 / 15)$ of patients who received interferon $\beta-1 \mathrm{~b}$ and azathioprine had elevated liver function tests which responded to a dose reduction, but none had serious complications [13]. On the other hand, in 2 other studies with 23 and 181 patients, 


\begin{tabular}{l|l}
\hline DOI: $10.1159 / 000354042$ & $\begin{array}{l}\text { C } 2013 \text { S. Karger AG, Basel } \\
\text { www.karger.com/crn }\end{array}$ \\
\hline
\end{tabular}

Liao et al.: Delayed Liver Function Impairment Secondary to Interferon $\beta$-1a Use in Multiple Sclerosis

respectively, who received interferon $\beta$-1a and azathioprine therapy, no abnormal liver function test was reported $[14,15]$. Although the results of previous studies remain controversial, it is still possible that concomitant use of azathioprine and interferon might have caused liver function impairment in patient 1.

In conclusion, our case reports show that interferon $\beta$-1a treatment in multiple sclerosis may lead to delayed liver function impairment several years later. Delayed interferon $\beta-1 \mathrm{a}-$ induced liver function abnormality is probably an idiosyncratic reaction and concomitant use of liver-toxic medication might play a role. Regular liver function test monitoring in multiple sclerosis patients who receive interferon $\beta$ treatment is necessary even after the first 6 months, especially in patients with concomitant use of liver-toxic medication.

\section{Disclosure Statement}

The authors have no conflicts of interest.

\section{References}

$\longrightarrow 1$ Andersen 0, Elovaara I, Farkkila M, Hansen HJ, Mellgren SI, Myhr KM, Sandberg-Wollheim M, Soelberg Sorensen P: Multicentre, randomised, double blind, placebo controlled, phase III study of weekly, low dose, subcutaneous interferon beta-1a in secondary progressive multiple sclerosis. J Neurol Neurosurg Psychiatry 2004;75:706-710.

2 PRISMS-4: Long-term efficacy of interferon-beta-1a in relapsing MS. Neurology 2001;56:1628-1636.

-3 PRISMS (Prevention of Relapses and Disability by Interferon beta-1a Subcutaneously in Multiple Sclerosis) Study Group: Randomised double-blind placebo-controlled study of interferon beta-1a in relapsing/remitting multiple sclerosis. Lancet 1998;352:1498-1504.

-4 Francis GS, Grumser Y, Alteri E, Micaleff A, O’Brien F, Alsop J, Stam Moraga M, Kaplowitz N: Hepatic reactions during treatment of multiple sclerosis with interferon-beta-1a: incidence and clinical significance. Drug Saf 2003;26:815-827.

5 Tremlett H, Seemuller S, Zhao Y, Yoshida EM, Oger JD, Petkau J: Liver test abnormalities in multiple sclerosis: findings from placebo-treated patients. Neurology 2006;67:1291-1293.

-6 Chan S, Kingwell E, Oger J, Yoshida E, Tremlett H: High-dose frequency beta-interferons increase the risk of liver test abnormalities in multiple sclerosis: a longitudinal study. Mult Scler 2011;17:361-367.

-7 Wingerchuk DM, Lennon VA, Pittock SJ, Lucchinetti CF, Weinshenker BG: Revised diagnostic criteria for neuromyelitis optica. Neurology 2006;66:1485-1489.

-8 Polman CH, Reingold SC, Banwell B, Clanet M, Cohen JA, Filippi M, Fujihara K, Havrdova E, Hutchinson M, Kappos L, Lublin FD, Montalban X, O'Connor P, Sandberg-Wollheim M, Thompson AJ, Waubant E, Weinshenker B, Wolinsky JS: Diagnostic criteria for multiple sclerosis: 2010 revisions to the McDonald criteria. Ann Neurol 2011;69:292-302.

19 Danan G, Benichou C: Causality assessment of adverse reactions to drugs - I. A novel method based on the conclusions of international consensus meetings: application to drug-induced liver injuries. J Clin Epidemiol 1993;46:1323-1330.

10 Christopher V, Scolding N, Przemioslo RT: Acute hepatitis secondary to interferon beta-1a in multiple sclerosis. J Neurol 2005;252:855-856.

11 Byrnes V, Afdhal N, Challies T, Greenstein PE: Drug induced liver injury secondary to interferon-beta (IFNbeta) in multiple sclerosis. Ann Hepatol 2006;5:56-59.

12 Bradley WG, Daroff RB, Fenichel GM, Jankovic J: Neurology in Clinical Practice. Philadelphia, ButterworthHeinemann, 2008, pp 2390-2391.

13 Pulicken M, Bash CN, Costello K, Said A, Cuffari C, Wilterdink JL, Rogg JM, Mills P, Calabresi PA: Optimization of the safety and efficacy of interferon beta $1 \mathrm{~b}$ and azathioprine combination therapy in multiple sclerosis. Mult Scler 2005;11:169-174.

14 Lus G, Romano F, Scuotto A, Accardo C, Cotrufo R: Azathioprine and interferon beta(1a) in relapsingremitting multiple sclerosis patients: increasing efficacy of combined treatment. Eur Neurol 2004;51:15-20.

-15 Havrdova E, Zivadinov R, Krasensky J, Dwyer MG, Novakova I, Dolezal O, Ticha V, Dusek L, Houzvickova E, Cox JL, Bergsland N, Hussein S, Svobodnik A, Seidl Z, Vaneckova M, Horakova D: Randomized study of interferon beta-1a, low-dose azathioprine, and low-dose corticosteroids in multiple sclerosis. Mult Scler 2009;15:965-976. 